\title{
Particulate matter and emergency visits for asthma: a time-series study of their association in the presence and absence of wildfire smoke in Reno, Nevada, 2013-2018
}

\section{Daniel Kiser}

Desert Research Institute

William J. Metcalf

Desert Research Institute

Gai Elhanan

Desert Research Institute

\section{Brendan Schnieder}

Washoe County Health District Air Quality Management Division

Karen Schlauch

Desert Research Institute

\section{Andrew Joros}

Desert Research Institute

\section{Craig Petersen}

Washoe County Health District Air Quality Management Division

Joseph Grzymski ( $\nabla$ joe.grzymski@dri.edu )

Desert Research Institute https://orcid.org/0000-0003-2646-8958

\section{Research}

Keywords: asthma, wildfires, particulate matter, smoke, emergency department, urgent care, Reno, Nevada, generalized additive model, interaction

Posted Date: August 18th, 2020

DOI: https://doi.org/10.21203/rs.2.21450/v2

License: (c) (i) This work is licensed under a Creative Commons Attribution 4.0 International License. Read Full License 
Version of Record: A version of this preprint was published on August 27th, 2020. See the published version at https://doi.org/10.1186/s12940-020-00646-2. 
1 Title: Particulate matter and emergency visits for asthma: a time-series study of their association in the

2 presence and absence of wildfire smoke in Reno, Nevada, 2013-2018

3

4 Authors: Daniel Kiser ${ }^{1,2}$, William J. Metcalf ${ }^{1,2}$, Gai Elhanan ${ }^{1,2}$, Brendan Schnieder ${ }^{3}$, Karen Schlauch ${ }^{1,2}$,

5 Andrew Joros ${ }^{1,2}$, Craig Petersen ${ }^{3}$, Joseph Grzymski ${ }^{1,2}$

6

7 Affiliations:

$8 \quad{ }^{1}$ Renown Institute for Health Innovation, Reno, Nevada, United States

$9 \quad{ }^{2}$ Department of Earth and Ecosystem Sciences, Desert Research Institute, Reno, Nevada, United States

$10{ }^{3}$ Washoe County Health District Air Quality Management Division, Reno, Nevada, United States

11

12 Corresponding Author: Joseph Grzymski

13 Email-joe.grzymski@dri.edu

14 Postal - 2215 Raggio Pkwy, Reno, NV 89512-1095

15

16 Other authors' email addresses:

17 Daniel Kiser-daniel.kiser@dri.edu

18 William J. Metcalf-jim.metcalf@dri.edu

19 Gai Elhanan-gai.elhanan@dri.edu 
21 Karen Schlauch - karen.schlauch@dri.edu

22 Andrew Joros - andrew.joros@dri.edu

23 Craig Petersen - cpetersen@washoecounty.us

24

25

26

27

28

29

30

31

32

33

34

35

36

37

38 
40 Background: Health risks due to particulate matter (PM) from wildfires may differ from risk due to PM

41 from other sources. In places frequently subjected to wildfire smoke, such as Reno, Nevada, it is critical

42 to determine whether wildfire PM poses unique risks. Our goal was to quantify the difference in the

43 association of adverse asthma events with PM on days when wildfire smoke was present versus days

44 when wildfire smoke was not present.

45 Methods: We obtained counts of visits for asthma at emergency departments and urgent care centers

46 from a large regional healthcare system in Reno for the years 2013-2018. We also obtained dates when

47 wildfire smoke was present from the Washoe County Health District Air Quality Management Division.

48 We then examined whether the presence of wildfire smoke modified the association of $\mathrm{PM}_{2.5}, \mathrm{PM}_{10-2.5}$,

49 and $\mathrm{PM}_{10}$ with asthma visits using generalized additive models. We improved on previous studies by

50 accounting for possible non-linearity in the association between PM concentration and asthma visits:

51 wildfire-smoke days where the PM concentration exceeded the maximum PM concentration on other

52 days were excluded.

53 Results: Air quality was affected by wildfire smoke on 188 days between 2013 and 2018 . We found that

54 the presence of wildfire smoke increased the association of a $5 \mu \mathrm{g} / \mathrm{m}^{3}$ increase in daily and three-day

55 averages of $\mathrm{PM}_{2.5}$ with asthma visits by $6.1 \%$ (95\% confidence interval $\left.(\mathrm{Cl}): 2.1-10.3 \%\right)$ and $6.8 \%(\mathrm{Cl}: 1.2-$

$5612.7 \%)$, respectively. Similarly, the presence of wildfire smoke increased the association of a $5 \mu \mathrm{g} / \mathrm{m}^{3}$

57 increase in daily and three-day averages of $\mathrm{PM}_{10}$ with asthma visits by $5.5 \%(\mathrm{Cl}: 2.5-8.6 \%)$ and $7.2 \%(\mathrm{Cl}$ :

58 2.6-12.0\%), respectively. We did not observe any significant increases in association for $\mathrm{PM}_{10-2.5}$ or for

59 seven-day averages of $\mathrm{PM}_{2.5}$ and $\mathrm{PM}_{10}$. 
60 Conclusions: Since we found significantly stronger associations of $\mathrm{PM}_{2.5}$ and $\mathrm{PM}_{10}$ with asthma visits

61 when wildfire smoke was present, our results suggest that wildfire PM is more hazardous than non-

62 wildfire PM for patients with asthma.

63

64

\section{Keywords}

65 asthma, wildfires, particulate matter, smoke, emergency department, urgent care, Reno, Nevada,

66 generalized additive model, interaction

67

\section{Introduction}

69 Studies have shown that particulate matter (PM) from wildfires can have an adverse effect on the health

70 of patients with asthma [1-5]. However, it is not yet clear whether wildfire PM is more or less harmful

71 for health conditions like asthma than PM from other sources, such as transportation and industry $[6,7]$.

72 Differences in the effects of wildfire and non-wildfire PM seem likely, since several in vitro studies [8-10]

73 and at least one study using live mice [11] have reported differences in biological and chemical

74 responses to wildfire and non-wildfire PM. There have also been several population health studies that

75 have explicitly compared the effects of wildfire and non-wildfire PM on patients with asthma [12-14].

76 While these studies generally found a stronger association of adverse asthma events with wildfire PM

77 than with non-wildfire PM, the differences were often not statistically significant. Furthermore, since

78 concentrations of PM tend to be much higher during wildfire events, it is possible that the differences

79 between wildfire and non-wildfire PM found in these studies were due to non-linearity in the

80 association between PM and asthma. 
81 An accurate understanding of the impacts of wildfire smoke on population health is critical if forest

82 management policies are to effectively mitigate those impacts. The need to improve such policies is especially urgent for population health in places like Reno, Nevada, where wildfire smoke events occur

84 regularly during the warm season. These events are currently expected to become more frequent in

85 Reno, as the mean amount of area burned each year in the region immediately west of Reno is predicted to increase by $78 \%$ from the period $1996-2005$ to the period $2046-2055$, due to climate

87 change [15].

Because of its frequent smoke exposures, Reno is ideal for studying the health effects of wildfire smoke. However, it is also suitable because it is home to a single regional healthcare system, Renown Health, that is estimated to receive $68.4 \%$ of the acute care cases in northern Nevada (Alan Herak, Director of Decision Support, Renown Health, personal communication, May 14, 2019; estimate based on statewide health billing data [16]). Because of its dominant market share, Renown's electronic health records (EHR) offer a comprehensive view of acute health problems resulting from wildfires occurring near Reno. Reno and neighboring Sparks (which we refer to collectively as Reno) have a combined population of approximately 355,000 [17] and are located in an intermountain desert valley at about 4,500 feet in elevation.

Only two previous studies have investigated the association of PM with asthma visits in Reno. In the first, Yang et al. [18] found no association between $\mathrm{PM}_{10}$ (PM between 0 and 10 micrometers in diameter) and emergency department (ED) visits. In the second, Rosenquist et al. [19] found associations between $\mathrm{PM}_{2.5}$ (PM less than 2.5 micrometers in diameter, or fine PM) and any type of asthma visit-such as office visits, ED visits, urgent care visits, or hospitalizations - with differences in association for allergic and nonallergic asthma patients. Neither Rosenquist et al. nor Yang et al. examined how the association of PM with asthma visits may be modified during wildfires. However, 
104 Rosenquist et al. speculated that they may have seen elevated risk associated with $\mathrm{PM}_{2.5}$ during the

105 warm season due to differences in the composition of wildfire $\mathrm{PM}_{2.5}$ compared to non-wildfire $\mathrm{PM}_{2.5}$.

106 Our goal in this study was to estimate the extent to which the presence of wildfire smoke changes the

107 association between PM and daily counts of asthma visits to EDs and UC centers in Reno, and hence to 108 investigate whether PM from wildfires is more or less hazardous for asthma patients than PM from 109 other sources.

\section{Methods}

112 Data

113 We obtained daily measurements of 24-hr average $\mathrm{PM}_{2.5}, 24-\mathrm{hr}$ average $\mathrm{PM}_{10-2.5}$ (PM between 2.5 and

11410 micrometers in diameter, or coarse PM), and 24-hr average PM 10 for the years 2013 through 2018

115 from four air quality monitors in the Reno area (Figure 1). We used only the measurements for each PM

116 fraction that were reported under local conditions to ensure consistency across measurements.

117 Population exposure to each fraction of PM was estimated using a weighted average of the monitors

118 that were active on each day. As weights, we used the counts of Renown patients whose last known

119 address was within a $5 \mathrm{~km}$ radius of each monitor (with the exception of the Galletti monitor, where we

120 used a radius of $2 \mathrm{~km}$ due to a nearby sandlot that affected the local air quality). Only one of the four

121 monitors was active for the entire study period for all three PM fractions.

122 We also obtained qualifier data, which is an hour by hour list of events that may have affected pollutant 123 data, from the Washoe County Health District Air Quality Management Division (WCHD-AQMD). These

124 events include monitor malfunctions, maintenance, or calibration, as well as the presence of smoke

125 from a wildfire or a prescribed burn. This data is submitted to the Environmental Protection Agency 
126 (EPA) quarterly and is subject to EPA audits. However, while every agency is required to record events

127 such as monitor maintenance and calibration, recording smoke events is voluntary, and thus data on

128 smoke events is not necessarily available from every air monitoring agency. WCHD-AQMD began

129 recording smoke events consistently at the beginning of 2013 , and these events were determined

130 primarily by the following criteria:

131

132

133

134

135

136

137

138

139

140

141

142

143

144

145

146 147 "non-wildfire days".

1) Elevated levels of organic carbon in speciated $\mathrm{PM}_{2.5}$

2) High concentrations of $\mathrm{PM}_{2.5}$ for the time of year (greater than $12 \mu \mathrm{g} / \mathrm{m}^{3}$ during the warm season was considered unusual)

3) High concentrations of $P M_{2.5}$ compared to recent data (i.e. a spike)

4) Deviations of $\mathrm{PM}_{2.5}$ from its typical diurnal pattern in the warm season

Other criteria that were used but which carried less weight than the above criteria were concentrations of $\mathrm{PM}_{10}, \mathrm{PM}_{10-2.5}$, nitrogen oxides, and ozone; whether nitrogen oxides and ozone deviated from their diurnal patterns; visibility at the Reno-Tahoe Airport; forecasts by the National Weather Service; the ratio of $\mathrm{PM}_{2.5}$ to $\mathrm{PM}_{10}$, with a ratio greater than 0.5 being considered indicative of wildfire smoke; the ratio of carbon monoxide to $\mathrm{PM}_{2.5}$; satellite images; and HYSPLIT (Hybrid Single Particle Lagrangian Integrated Trajectory) models.

For our analysis, we did not make a distinction between smoke from a wildfire and smoke from a prescribed burn, and we considered the air quality on any given day to be affected by wildfire smoke if any hour of that day was affected, as indicated by the qualifier data from the Reno3 air quality monitor (which is located in downtown Reno). We henceforth refer to days where the air quality was affected by wildfire smoke as "wildfire days" and days where the air quality was not affected by wildfire smoke as 
148 Hourly temperature and humidity data were obtained from the KRNO weather station located at the 149 Reno-Tahoe Airport (near downtown Reno). We calculated the daily minimum, mean, and maximum 150 values for temperature, and the daily mean for relative humidity (RH). Visits for asthma were defined as

151 hospital or office visit encounters with an associated diagnosis code matching ICD-10 codes J45.20-

$152 \quad J 45.998$ and/or ICD-9 codes 493.00-493.92. Counts of visits for the years 2013-2018 were obtained from

153 Renown's EHR for two EDs and seven UC centers (Figure 1). We had full access to Renown's EHR for the 154 time period under investigation.

155

\section{Analyses}

For modeling, we used a generalized additive model (GAM) from the Negative Binomial family, a distribution family which allows for overdispersed Poisson outcomes. To construct our models, we first created a base model that had the following form:

$$
\log (E \text { (visits }))=\alpha+\gamma D O W+\text { Sholidays }+\operatorname{cr}(\text { time })+\zeta \text { temp }+\eta A R
$$

where DOW represents six separate indicator variables for days of the week, holidays represents 17 separate indicator variables for selected holidays, $\operatorname{cr}($ time) represents a cubic regression spline of time, temp represents a rolling seven-day average of daily mean temperature, and $A R$ represents autoregressive terms introduced to control for autocorrelation. After examining autocorrelation of the base model residuals using autocorrelation function (ACF) and partial ACF plots, we added autoregressive terms for lags 1-10 (lag 1 of asthma visits, lag 2 of asthma visits, etc.). Degrees of freedom for $\operatorname{cr}($ time $)$ were not selected a priori, since the R package used for building the GAMs, mgcv, uses a penalized regression spline approach that automatically determines the degree of smoothness [20].

In order to examine both immediate and delayed associations between asthma visits and PM, we constructed variables for each $\mathrm{PM}$ fraction $\left(\mathrm{PM}_{2.5}, \mathrm{PM}_{10-2.5}\right.$, and $\left.\mathrm{PM}_{10}\right)$ representing immediate exposure 
171 (lag 0), short-term cumulative exposure (rolling average of lags 0-2), and longer-term cumulative

172 exposure (rolling average of lags $0-6$ ). We similarly constructed indicator variables for days affected by

173 wildfire smoke: an indicator for at least one wildfire day (lag 0), an indicator for at least three wildfire

174 days in a row (lags 0-2), and an indicator for at least seven wildfire days in a row (lags 0-6). The last two

175 wildfire indicators had the value " 1 " when all of the included lags were wildfire days, but " 0 " only when

176 none of the included lags were wildfire days. If the lags were a mix of wildfire days and non-wildfire

177 days, we excluded that observation from the analysis whenever that indicator was used. In our models

178 described below, we always use the indicator variable for wildfires whose lags correspond to the lags

179 used to construct the PM variable (lags 0, 0-2, and 0-6). Finally, we also constructed variables indicating

180 the daily activity status of each air quality monitor, to account for differences in air quality at each

181 monitoring site. For models that included lag 0-2 and lag 0-6 averages of PM, these variables indicated

182 whether a particular monitor was active at any lag on any given day.

Because of the possibility of a nonlinear association between PM and asthma visits (such as a stronger

184 association at higher concentrations of PM than at lower concentrations), we excluded wildfire days if

185 the concentration of $\mathrm{PM}_{2.5}$ on those days exceeded the maximum $\mathrm{PM}_{2.5}$ concentration observed on non-

186 wildfire days. This was done separately for each set of lags, as a high value of $\mathrm{PM}_{2.5}$ on one day would

187 not necessarily cause a rolling average of $\mathrm{PM}_{2.5}$ concentrations on wildfire days to exceed the maximum

188 of the rolling average on non-wildfire days. We only excluded days on the basis of $\mathrm{PM}_{2.5}$, and not $\mathrm{PM}_{10 \text { - }}$

1892.5 or $\mathrm{PM}_{10}$, since the maximum concentration of $\mathrm{PM}_{10-2.5}$ was higher on non-wildfire days than on

190 wildfire days.

191 For our analyses, the values of $\mathrm{PM}_{2.5}, \mathrm{PM}_{10-2.5}$, and $\mathrm{PM}_{10}$ were scaled so that a unit change is equivalent

192 to $5 \mu \mathrm{g} / \mathrm{m}^{3}$, and we defined statistical significance as $p<0.05$. In order to determine whether PM is

193 more hazardous on wildfire days than on non-wildfire days, we modeled an interaction between PM and 194 wildfires as follows: 
$\log ($ E(visits $))$

$$
\begin{aligned}
& =\boldsymbol{\alpha}+\boldsymbol{\gamma} \boldsymbol{D O W}+\boldsymbol{\delta h o l i d a y s}+\boldsymbol{c r}(\text { time })+\zeta \text { temp }+\boldsymbol{\eta} \boldsymbol{A R}+\beta_{1} P M \\
& +\beta_{2}(P M \times W F)+\beta_{3} W F+\theta M O N_{P M}
\end{aligned}
$$

195 where the terms in bold belong to the base model. PM represents any of the three fractions of PM $196\left(\mathrm{PM}_{2.5}, \mathrm{PM}_{10-2.5}\right.$, or $\left.\mathrm{PM}_{10}\right)$ from any of the three lag sets (lags $0,0-2$, or $0-6$ ), while $W F$ represents the 197 corresponding wildfire indicator from the same lag set. $M O N_{P M}$ represents the set of indicator variables 198 for the activity status of each monitor. We constructed nine different models of this form, one for each 199 combination of PM fraction and lag sets.

200 In these models, which we refer to as the "single-fraction models", the rate ratio for the change in the 201 number of asthma visits due to a $5 \mu \mathrm{g} / \mathrm{m}^{3}$ change in PM is given by: $\exp \left(\widehat{\beta_{1}}+\widehat{\beta_{2}} W F\right)$, where $\exp \left(\widehat{\beta_{1}}\right)$ is 202 the rate ratio when wildfire smoke is not present (i.e., $W F=0)$ and $\exp \left(\widehat{\beta_{1}}+\widehat{\beta_{2}}\right)$ is the rate ratio 203 when wildfire smoke is present (i.e, $W F=1) \cdot \exp \left(\widehat{\beta_{2}}\right)$ quantifies the multiplicative change in the rate 204 ratio when wildfire smoke is present, and allows us to construct confidence intervals for this change.

205 We also report this change as a percent: $\left(\exp \left(\widehat{\beta_{2}}\right)-1\right) \times 100$. To estimate the variance of $\beta_{1}+\beta_{2}$, 206 we used the standard formula for the variance of a linear combination of random variables, similar to 207 Deflorio-Barker et al. [12].

208 We also created an additional model, which we refer to as the "two-fraction model", that allowed us to 209 predict the increase in asthma visits due to the presence of wildfire smoke for any given levels of $\mathrm{PM}_{2.5}$ 210 and $\mathrm{PM}_{10-2.5}$. This model included terms for both lag- $0 \mathrm{PM}_{2.5}$ and $\mathrm{PM}_{10-2.5}$ and their respective 211 interactions with the wildfire indicator: 


$$
\begin{array}{r}
\log (\boldsymbol{E}(\text { visits }))=\boldsymbol{\alpha}+\boldsymbol{\gamma} \boldsymbol{D O W}+\boldsymbol{\delta h o l i d a y s}+\boldsymbol{c r}(\boldsymbol{t i m e})+\zeta \text { temp }+\boldsymbol{\eta} \boldsymbol{A R}+\beta_{1} P M_{2.5}+ \\
\beta_{2}\left(P M_{2.5} \times W F\right)+\beta_{3} P M_{10-2.5}+\beta_{4}\left(P M_{10-2.5} \times W F\right)+\beta_{5} W F+\theta M O N_{P M 2.5}+ \\
\lambda M O N_{P M 10-2.5}
\end{array}
$$

212 In contrast to equation (1), the $P M$ and $W F$ terms in equation (2) indicate only lag 0 constructions,

213 while $M O N_{P M 2.5}$ and $M O N_{P M 10-2.5}$ represent sets of indicators for monitors actively measuring $\mathrm{PM}_{2.5}$

214 and $\mathrm{PM}_{10-2.5}$, respectively. The estimated percent increase in asthma visits due to the presence of

215 wildfire smoke at given levels of $\mathrm{PM}_{2.5}$ and $\mathrm{PM}_{10-2.5}$ (scaled to $5 \mu \mathrm{g} / \mathrm{m}^{3}$ ) was calculated as:

$$
\left(\exp \left(\widehat{\beta_{2}} P M_{2.5}+\widehat{\beta_{4}} P M_{10-2.5}+\widehat{\beta_{5}}\right)-1\right) \times 100
$$

216 The variance of the expression $\beta_{2} P M_{2.5}+\beta_{4} P M_{10-2.5}+\beta_{5}$, which was used to calculate $p$-values, was 217 calculated using the standard formula for the variance of a linear combination of random variables, as 218 follows: $P M_{2.5}$ and $P M_{10-2.5}$

To test the stability of the models, we repeated the analysis using different controls for meteorological factors. Specifically, we replaced the rolling seven-day average of daily mean temperature with a rolling seven-day average of either daily maximum temperature or daily minimum temperature. We also repeated the analysis using a rolling seven-day average of daily mean $\mathrm{RH}$ in addition to our control for daily mean temperature. We then compared the results of the sensitivity analyses to that of the 
primary analysis. We used R version 3.6.0 and the R packages mgcv 1.8-28 [20], mgcv.helper 0.1.8 [21], and TSA 1.2 [22] for all of our statistical modeling, and we used SAS 9.4 for extracting data from Renown's electronic health records. Work on this study was a part of a larger body of work, "Interoperability, operational efficiency and quality of care improvements through health data analysis."

\section{Results}

234 Air quality was affected by wildfire smoke on 188 days between 2013 and 2018 (Figure 2). Of these 235 days, there were 125 days that were preceded by at least two other wildfire days (lags 0-2), and 68 days that were preceded by at least six other wildfire days (lags 0-6). Although we defined wildfire days as

237 days where the air quality was affected by wildfire smoke for at least one hour, air quality on wildfire 238 days was generally affected by smoke for most of the day, with 182 days affected by smoke for more than 20 hours and only 6 days affected by smoke for less than 20 hours (Figure S1). Inspection of the data revealed that small decreases in the number of hours recorded as affected by wildfire smoke on 241 any given day were often caused by brief periods of monitor malfunction or maintenance. We found 242 that the maximum levels of $\mathrm{PM}_{2.5}$ for non-wildfire days were $40.4 \mu \mathrm{g} / \mathrm{m}^{3}$ (lag 0), $36.8 \mu \mathrm{g} / \mathrm{m}^{3}$ (rolling 243 average of lags $0-2$ ), and $35.0 \mu \mathrm{g} / \mathrm{m}^{3}$ (rolling average of lags $0-6$ ). After excluding wildfire days where $244 \mathrm{PM}_{2.5}$ exceeded these maximums, we retained 175 solitary wildfire days, 107 wildfire days where the 245 previous two days were also wildfire days, and 50 wildfire days where the previous six days were also 246 wildfire days. The most severe wildfire smoke events, with the highest $\mathrm{PM}_{2.5}$ levels and the longest 247 duration, occurred in August-September of 2013, September of 2014, and July-September of 2018 248 (Figure 2).

As expected, concentrations of $\mathrm{PM}_{10}$ were highest on days affected by wildfire smoke, with mean 250 concentrations of $\mathrm{PM}_{10}$ increasing from $17.9 \mu \mathrm{g} / \mathrm{m}^{3}$ during non-wildfire periods to $31.2 \mu \mathrm{g} / \mathrm{m}^{3}$ during 251 wildfire periods (Table 1). However, most of that increase was due to $\mathrm{PM}_{2.5}$, for which mean 
252

253

254

255

256

257

258

259

260

261

262

263

264

265

266

267

268

269

270

271

272

273

274

275

concentrations increased from $7.1 \mu \mathrm{g} / \mathrm{m}^{3}$ during the non-wildfire period to $19.4 \mu \mathrm{g} / \mathrm{m}^{3}$ during the wildfire period, while mean concentrations of $\mathrm{PM}_{10-2.5}$ only increased from $10.9 \mu \mathrm{g} / \mathrm{m}^{3}$ to $11.6 \mu \mathrm{g} / \mathrm{m}^{3}$. In fact, maximum concentrations of $\mathrm{PM}_{10-2.5}$ were higher during non-wildfire periods (Table 1). The variation in $\mathrm{PM}_{10-2.5}$ was also lower on wildfire days than on non-wildfire days, with the standard deviation (SD) decreasing from 7.1 to 4.8 , in contrast to the SDs for $\mathrm{PM}_{2.5}$ and $\mathrm{PM}_{10}$, which increase from 4.8 to 14.8 and from 10.6 to 16.5 , respectively. When we examine the Pearson correlations among the different size fractions of PM (Table 2), we note that concentrations of $\mathrm{PM}_{10}$ were correlated with both $\mathrm{PM}_{2.5}$ and $\mathrm{PM}_{10-2.5}$ on non-wildfire days ( 0.87 and 0.94 , respectively), but that during wildfires $\mathrm{PM}_{10}$ was more correlated with $\mathrm{PM}_{2.5}$, with a correlation of 0.96 , than with $\mathrm{PM}_{10-2.5}$, with a correlation of 0.46 .

There were 18,836 ED and UC center visits for asthma between 2013 and 2018. The average number of daily visits for asthma were slightly higher on non-wildfire days than on wildfire days (8.6 versus 8.2 visits, Table 1). This small decrease in daily visits on wildfire days can be attributed to a generally higher number of daily visits during the cold season than during the warm season (Figure 2), when wildfires are likely to occur.

In the single-fraction models (see equation 1), we found that PM had a positive association with asthma visits for all combinations of lags and PM fractions on non-wildfire days (Figure 3). However, with the exception of lag 0-6 $\mathrm{PM}_{10-2.5}$, rate ratios were larger on wildfire days. This increase in rate ratio was significant for $\mathrm{PM}_{2.5}$ and $\mathrm{PM}_{10}$ at lag 0 and lag $0-2$. The rate ratio for a $5 \mu \mathrm{g} / \mathrm{m}^{3}$ change in lag-0 $\mathrm{PM} 2.5$ increased from 1.007 (Cl: 0.988-1.026) to 1.068 (Cl: 1.033-1.105), an increase of 6.1\% (Cl: 2.1-10.3\%), while the rate ratio for lag 0-2 $\mathrm{PM}_{2.5}$ increased from 1.014 (Cl: $\left.0.992-1.037\right)$ to 1.083 (Cl: 1.031-1.138), an increase of $6.8 \%$ ( $\mathrm{Cl}: 1.2-12.7 \%)$. Similarly, the rate ratio for a $5 \mu \mathrm{g} / \mathrm{m}^{3}$ change in lag-0 $\mathrm{PM}_{10}$ increased from 1.006 ( $\mathrm{Cl}: 0.998-1.015)$ to 1.062 ( $\mathrm{Cl}: 1.032-1.092)$, an increase of $5.5 \%$ ( $\mathrm{Cl}: 2.5-8.6 \%)$, while the rate ratio for lag 0-2 $\mathrm{PM}_{10}$ increased from 1.009 (Cl: 0.999-1.019) to 1.082 (Cl: 1.036-1.130), an increase of 7.2\% (Cl: 2.6-12.0\%). We did not observe any significant increases in association for $\mathrm{PM}_{10-2.5}$ or for lag 0- 
$6 \mathrm{PM}_{2.5}$ and $\mathrm{PM}_{10}$. Confidence intervals for $\mathrm{PM}_{10-2.5}$ on wildfire days were much wider than the confidence intervals for either $\mathrm{PM}_{2.5}$ or $\mathrm{PM}_{10}$ on wildfire days. It is important to emphasize that the magnitude of the changes in association described above varies with the unit scale used for PM, even though the statistical significance of the changes remains the same.

The results of the two-fraction model (see equation 2) are visualized in Figure 4. Instead of examining the association between PM and asthma visits and how it changes in the presence of wildfire smoke, as in Figure 3, we here examine the association between wildfire smoke and asthma visits and how it changes at different levels of PM. The estimated percent change in asthma visits due to the presence of wildfire smoke is $\left(\exp \left(0.0747 \times P M_{2.5}+0.0341 \times P M_{10-2.5}-0.2259\right)-1\right) \times 100$, where $\mathrm{PM}_{2.5}$ and $\mathrm{PM}_{10-2.5}$ are scaled to $5 \mu \mathrm{g} / \mathrm{m}^{3}$ (equation 3). Thus, we would expect the presence of wildfire smoke to increase the number of asthma visits by $19.1 \%$ when $\mathrm{PM}_{2.5}$ is at $20.0 \mu \mathrm{g} / \mathrm{m}^{3}$ and $\mathrm{PM}_{10-2.5}$ is at $15.0 \mu \mathrm{g} / \mathrm{m}^{3}$ ( $p=0.00092$ ). If both $\mathrm{PM}_{2.5}$ and $\mathrm{PM}_{10-2.5}$ increased by $5.0 \mu \mathrm{g} / \mathrm{m}^{3}$, to 25.0 and $20.0 \mu \mathrm{g} / \mathrm{m}^{3}$ respectively, we would then expect the presence of wildfire smoke to increase the number of asthma visits by $32.8 \%(p=$ 0.00022). In addition to limiting extrapolation of the model results, the overlaid scatterplot of observed values in Figure 4 also illustrates the difference in the $\mathrm{PM}_{2.5} / \mathrm{PM}_{10-2.5}$ ratio on wildfire days and nonwildfire days. The mean $\mathrm{PM}_{2.5} / \mathrm{PM}_{10-2.5}$ ratio was 1.62 on wildfire days and 0.77 on non-wildfire days (after excluding wildfire days where $\mathrm{PM}_{2.5}$ exceeded the maximum $\mathrm{PM}_{2.5}$ of non-wildfire days).

The results of the sensitivity analyses (shown in the supplementary materials) were nearly identical to the results of the primary analysis. However, estimates for non-wildfire days in our single-fraction models were slightly depressed when daily mean temperature was replaced with daily minimum temperature, and they were slightly inflated when daily mean temperature was replaced with daily maximum temperature (Tables S1, S2, S3 and Figures 3, S2, S3). These changes in the estimates for nonwildfire days influenced the estimates for the change in association due to wildfires as well, such that estimates in the models controlling for daily minimum temperature were somewhat increased while the 
estimates in the models controlling for daily maximum temperature decreased. For example, the lag 0

301

302

estimates for $\mathrm{PM}_{2.5}$ on non-wildfire days in models controlling for minimum, mean, and maximum temperature were 1.003 (Cl: 0.984-1.022), 1.007 (Cl: 0.988-1.026), 1.013 (Cl: 0.995-1.032), respectively; while the lag 0 estimates for the increase in the association of PM due to the presence of wildfire smoke were $6.6 \%$ (Cl: $2.5-10.8 \%), 6.1 \%$ (Cl: $2.1-10.3 \%), 5.1 \%$ (Cl: $1.2-9.2 \%)$, respectively. No meaningful differences in the primary results were found when a seven-day rolling average of daily mean RH was included in the model in addition to daily mean temperature (Tables S1, S4 and Figures 3, S4), nor were there meaningful differences in the results of the two-fraction model in any of the sensitivity analyses (Table S5 and Figures 4, S5-S7).

\section{We found that the presence of wildfire smoke increases the association of $\mathrm{PM}_{2.5}$ and $\mathrm{PM}_{10}$ with asthma} visits at shorter lags (lag 0 and lags $0-2$ ). We did not find a significant increase in association for $\mathrm{PM}_{10-2.5}$ at any lag, nor did we find a significant increase in association at lag 0-6 for any PM fraction.

The similarity between estimates for $\mathrm{PM}_{2.5}$ and $\mathrm{PM}_{10}$ when wildfire smoke was present was probably due to the high correlation (0.96) between $\mathrm{PM}_{2.5}$ and $\mathrm{PM}_{10}$ on wildfire days, which suggests that a change in wildfire $\mathrm{PM}_{10}$ was roughly equivalent to a change in wildfire $\mathrm{PM}_{2.5}$. It is possible that we did not see a significant increase in association at lags 0-6 for any PM fraction because there were two few wildfire days preceded by six other consecutive wildfire days-only 50 days, compared to 107 days for lag 0-2 and 175 days for lag 0 -thus making our sample size too small. Similarly, we may not have detected a significant association of $\mathrm{PM}_{10-2.5}$ with asthma visits on wildfire days because the variation of $\mathrm{PM}_{10-2.5}$ on wildfire days (SD: 4.8) was much lower than that of $\mathrm{PM}_{2.5}$ (SD: 14.8) and $\mathrm{PM}_{10}$ (SD: 16.5). It is worth noting that the lower Cls for wildfire $\mathrm{PM}_{10-2.5}$ at lag 0 and lags $0-2$ were quite close to excluding a rate ratio of 1.0 , suggesting that a larger sample size might be able to detect a significant association 
323 between $\mathrm{PM}_{10-2.5}$ on wildfire days (and possibly an increase in association compared to non-wildfire

324 days).

325 Our study used models very similar to those of Deflorio-Barker et al. [12], Reid et al. [13], and Delfino et

326 al. [14], which also included an interaction term between PM and an indicator for days affected by

327 wildfire smoke. However, a key difference in our study is that we excluded wildfire days where $\mathrm{PM}_{2.5}$

328 concentrations exceeded the maximum $\mathrm{PM}_{2.5}$ concentration of non-wildfire days. We believe that this is

329 important, since the association of wildfire PM with asthma may be stronger at higher concentrations of

330 PM than at lower concentrations $[3,13]$. By eliminating wildfire days with excessively high $\mathrm{PM}_{2.5}$

331 concentrations (and, by extension, excessively high $\mathrm{PM}_{10}$ concentrations), we ensured that the stronger

332 associations we found for wildfire PM compared to non-wildfire PM could not be attributed to a

333 nonlinear association between PM and asthma visits.

334 Despite removing wildfire days with extremely high $\mathrm{PM}_{2.5}$ concentrations, our results were similar to

335 those of Deflorio-Barker et al. [12], Reid et al. [13], and Delfino et al. [14], who all found that PM 2.5 had a

336 stronger association with asthma visits or hospitalizations during wildfire periods than during non-

337 wildfire periods. We thus showed that the stronger associations of wildfire PM with asthma visits found

338 in these studies cannot be solely attributed to differences in the concentration of wildfire PM and non-

339 wildfire PM. Furthermore, since there were mixed results from these studies regarding whether there

340 was a statistically significant increase in the association of $\mathrm{PM}_{2.5}$ with asthma during wildfires, our study

341 added further support to the hypothesis that the observed increases were not due to random variation.

342 Our results were especially meaningful since we contrasted the associations of non-wildfire PM and

343 wildfire PM at relatively low concentrations (i.e., at or below $40.4 \mu \mathrm{g} / \mathrm{m}^{3} \mathrm{PM}_{2.5}$ for lag 0 analyses), when

344 the association of PM with asthma visits would be expected to be less clear. We also examined a

345 relatively long time period of six years that included 175 days where the air quality was affected by

346 wildfire smoke. In contrast, the study by DeFlorio-Barker et al. [12] examined 3 years, while Reid et al. 
347 [13] and Delfino et al. [14] examined single wildfire smoke events. However, the study by DeFlorio-

348 Barker et al. covered the United States, rather than just one city, and it is likely that their estimates

349 represented the effects of PM on asthma patients over a much broader range of wildfire fuelbed types

350 and burn conditions than our study.

351 During our study period, air quality in Reno was affected by major wildfires from the Klamath, Northern

352 Coast, and Sierra Nevada mountain ranges. Using BlueSky Playground v3.0

353 (https://tools.airfire.org/playground/v3/emissionsinputs.php), we determined that the top five Fuel

354 Characteristic Classification System fuelbed types of these wildfires were mixed Jeffery pine forest,

355 mixed Douglas fir forest, red fir forest, oak woodlands, and chamise chaparral shrublands. Since fuelbed

356 types are unique to every region, our results may not be generalizable to regions where fuelbed types

357 are very different, such as the Southeastern United States.

The mechanisms which could make wildfire PM more or less harmful than non-wildfire PM for patients

359 with asthma are likely to be complex, but there are at least three broad categories into which these

360 mechanisms may fall: (1) differences in the composition of wildfire and non-wildfire PM, (2) interactions

361 between PM and temperature, and (3) patient behavioral changes. These mechanistic categories are

362 not mutually exclusive, and it is possible that each of them may play an important role in the

363 harmfulness of wildfire PM.

Perhaps the best supported mechanism for the increased harmfulness of wildfire PM is a change in PM

365 composition during wildfire events. The effects of wildfire PM on respiratory tissue has been compared

366 with that of non-wildfire PM in several laboratory studies. Nakayama Wong et al. [9] and Verma et al.

367 [10] showed that cell-culture response to PM from wildfires differed from that of non-wildfire PM,

368 though it was not clear in either of these studies whether one was more harmful than the other [23].

369 However, other studies have suggested that PM from wildfires is much more harmful than non-wildfire 
PM. In a study using live mice, Wegesser et al. [11] estimated that $\mathrm{PM}_{2.5}$ and $\mathrm{PM}_{10-2.5}$ from wildfires was

371 about 10 times as toxic as an equivalent mass of non-wildfire PM. Similarly, Franzi et al. [8] conducted

372 an in vitro study on PM from the same wildfire as Wegesser et al. and found that coarse PM (defined as

373 PM between 10.2-2.1 micrometers in diameter) from the wildfire was approximately four times as toxic

374 as non-wildfire coarse PM. It is not clear whether the results of Wegesser et al. and Franzi et al. are

375 generalizable to all wildfires, since Kim et al. [24] reported that the toxicity of PM from wildfires

376 depends on the activity status of the wildfire (flaming or smoldering) and the fuel type. Thus, more

377 research is needed to determine the conditions under which PM from wildfires is the most toxic, but it

378 at least seems that wildfire PM is more toxic than non-wildfire PM under some conditions.

A second possible mechanism for an increased $\mathrm{PM}_{2.5}$ association with asthma during wildfires is a $\mathrm{PM}$ -

temperature interaction, since wildfire events tend to be associated with higher ambient temperatures

[25]. Shaposhnikov et al. [26] found a statistically significant interaction between temperature and $\mathrm{PM}_{10}$

382 during a combined heat-wave/wildfire event in Moscow, Russia. Other studies have reported that

temperature modifies the effect of non-wildfire PM as well [27-30]. Part of the modifying effect of temperature may be mediated through changes in PM composition, so it is not possible to make a clear

PM on patients with asthma by inducing behavioral changes, since more people are likely to be outside

$\mathrm{PM}_{2.5}$ compared to non-wildfire $\mathrm{PM}_{2.5}$ that we observed in our study. 
speculated that this was due to older children effectively managing their symptoms using rescue

medications. Another study found that prescriptions fills for rescue medications increased during smoke events [33]. Both of these studies support the notion that patients with asthma are likely to take proactive action during a wildfire smoke event that reduces the risk of an ED or UC visit. Since our study uses ED and UC visits as health endpoints, such behavior could cause wildfire PM to appear less harmful in relation to PM from other sources.

Other than the composition of wildfire PM and possible PM-temperature interactions, the harmfulness of wildfire smoke may also be affected by particle size. In general, there is stronger evidence for the

402 harmfulness of $\mathrm{PM}_{2.5}$ than $\mathrm{PM}_{10-2.5}$ [34], regardless of whether or not the source is a wildfire. However, studies comparing the toxicity of wildfire $\mathrm{PM}_{2.5}$ and $\mathrm{PM}_{10-2.5}$ have produced mixed results [23]. Leonard et al. [35] concluded that ultrafine particles were the most reactive (and hence more harmful), while Jalava et al. [36] found that the toxicity of the different size fractions depended on how toxicity was measured. Dong et al. [23] attributed the differences between these two studies to differences in the

407 distance the PM was transported by the wind before it was collected. Wegesser et al. [11] found equal 408 toxicity for both $\mathrm{PM}_{2.5}$ and $\mathrm{PM}_{10-2.5}$, but noted that real-world exposure mechanisms might produce 409 different levels of toxicity in practice. The results of our study could be construed as support for the 410 hypothesis that finer wildfire PM is more hazardous for patients with asthma. However, the lack of a 411 positive modification of $\mathrm{PM}_{10-2.5}$ by wildfires in our study may also be attributed to the very small

412 increase in mean $\mathrm{PM}_{10-2.5}$ levels during wildfires over non-wildfire periods (Table 1), which suggests that 413 most of the $\mathrm{PM}_{10-2.5}$ observed during wildfire periods is coming from non-wildfire sources. Thus, in 414 addition to more research on PM composition, more research is needed on the relative toxicity of 415 different size fractions of PM.

416 It may also be worthwhile to investigate the role that the $\mathrm{PM}_{2.5} / \mathrm{PM}_{10-2.5}$ ratio plays in the harmfulness of 417 wildfire smoke, since the ratio differs between wildfire days and non-wildfire days (Figures 2 and 4). 
418 This possibility does not appear to have been investigated yet. Future work could also focus on

419 differences in the health effects of smoke from a prescribed burn versus a wildfire. In our study, no

420 distinction was made between prescribed burns and wildfires, but differences in the relative toxicity of

421 the smoke produced could have important implications for forest management policy.

422 Our study had several limitations. The most important of these was not being able to directly

423 distinguish between PM from wildfires and PM from other sources. Since we instead compared the

424 association of PM with asthma visits on days affected by wildfire smoke and days not affected, our study

425 likely underestimated the difference in association between wildfire PM and non-wildfire PM. We were

426 also limited by the necessity of dropping extremely high $\mathrm{PM}_{2.5}$ wildfire days in order to make an

427 appropriate comparison with non-wildfire days. Other than during the intermittent wildfire event,

428 levels of air pollution were relatively low in Reno, so a study like ours would perhaps be more effective

429 in a city with higher levels of non-wildfire PM. A third limitation, which applies to most ecological

430 studies like ours, was not being able to assess individual exposure levels. Although we combined data

431 from four different air monitors throughout Reno, exposures may vary a great deal depending on where

432 individuals live and work, and depending on the amount of time they spend outdoors.

433 A key strength of our study was that it spanned six years and included large and small wildfire events, in 434 contrast to many studies which examined a single large wildfire event. Thus, our data included wildfires 435 that occurred under a variety of conditions and at a wide range of distances from Reno, making our 436 estimates robust to variability between fires.

\section{Conclusions}

439 The main conclusion of this study was that PM from wildfires can be more harmful for patients with 440 asthma than PM from other sources, as we found statistically significant increases in the association of 
$441 \quad \mathrm{PM}_{2.5}$ and $\mathrm{PM}_{10}$ with emergency visits for asthma on wildfire days versus non-wildfire days. This

442 validated previous studies which found similar results but were not able to consistently detect a

443 statistically significant difference. Another important contribution of our study was that we showed that

444 the stronger association of wildfire PM with asthma visits cannot be solely attributed to higher

445 concentrations of PM on wildfire days versus non-wildfire days, since the effects of wildfire and non-

446 wildfire PM were compared at similar concentrations, and these concentrations were relatively low

447 compared to the concentrations that often occur during a wildfire smoke event. Thus, there appears to

448 be factors other than increased PM concentration that contribute to the increased harmfulness of

449 wildfire smoke. More research is needed to understand these factors, but current research suggests

450 that the harmfulness of wildfire PM may be affected by fuel source and burn rate [24], ambient

451 temperature [26], behavioral changes [31-33], and smoke transport distance [23].

\section{List of abbreviations}

453 ACF: autocorrelation function; $\mathrm{Cl}$ : confidence interval; ED: emergency department; EHR: electronic

454 health records; EPA: Environmental Protection Agency; GAM: generalized additive model; HYSPLIT:

455 Hybrid Single Particle Lagrangian Integrated Trajectory; PM: particulate matter; PM $_{2.5}$ : fine particulate

456 matter (0-2.5 micrometers in diameter); $\mathrm{PM}_{10}$ : particulate matter (0-10 micrometers in diameter); $\mathrm{PM}_{10-}$

457 2.5: coarse particulate matter (2.5-10 micrometers in diameter); RH: relative humidity; SD: standard

458 deviation; UC: urgent care; WCHD-AQMD: Washoe County Health District Air Quality Management

459 Division 
463 This study was exempted by the Institutional Review Board at the University of Nevada, Reno (886489-

464 2), since the human subject data used was anonymized and aggregated.

465 Consent for publication

466 Not applicable

467 Availability of data and materials

468 The datasets used and/or analyzed during the current study are available from the corresponding author

469 on reasonable request. In addition, air quality data is publicly available from the EPA's internet database 470 at https://www.epa.gov/airdata [37].

\section{Competing interests}

472 The authors declare that they have no competing interests.

\section{$473 \quad$ Funding}

474 Funding was provided by the Nevada Governor's Office of Economic Development, Renown Health and

475 the Renown Health Foundation. Renown Health provided data that was used in the study.

\section{Authors' contributions}

477 DK contributed to the design of the study, obtained air quality data, extracted data from the electronic 478 health records, conducted the statistical analyses, interpreted the results, and drafted the manuscript.

479 WM contributed to the design of the study, obtained air quality data, extracted data from the electronic 480 health records, and revised the manuscript. GE contributed to the design of the study, extracted data 481 from the electronic health records, and revised the manuscript. BS contributed to the design of the 
482 study and revised the manuscript. AJ obtained meteorological data and revised the manuscript. KS, CP,

483 and JG revised the manuscript. All authors read and approved the final manuscript.

\section{Acknowledgements}

We would like to give special thanks to Matthew Strickland (University of Nevada, Reno) for his guidance on the modeling aspects of our work and for his constructive criticism of the manuscript. We would also like to thank Dr. Debra Hendrickson (University of Nevada School of Medicine) for sharing her inputs and comments, and David Miceli (University of Nevada, Reno) for his help with obtaining spatial data.

\section{References}

1. Alman BL, Pfister G, Hao H, Stowell J, Hu X, Liu Y, et al. The association of wildfire smoke with respiratory and cardiovascular emergency department visits in Colorado in 2012: a case crossover study. Environ Heal [Internet]. 2016;15(1):64. Available from: https://doi.org/10.1186/s12940-016-0146-8

2. Haikerwal A, Akram M, Sim MR, Meyer M, Abramson MJ, Dennekamp M. Fine particulate matter (PM2.5) exposure during a prolonged wildfire period and emergency department visits for asthma. Respirology [Internet]. 2016 Jan 1;21(1):88-94. Available from: https://doi.org/10.1111/resp.12613

3. Johnston FH, Kavanagh AM, Bowman DMJS, Scott RK. Exposure to bushfire smoke and asthma: an ecological study. Med J Aust [Internet]. 2002 Jun 1;176(11):535-8. Available from: https://doi.org/10.5694/j.1326-5377.2002.tb04551.x

4. Künzli N, Avol E, Wu J, Gauderman WJ, Rappaport E, Millstein J, et al. Health effects of the 2003 Southern California wildfires on children. Am J Respir Crit Care Med [Internet]. 2006/08/31. 2006 Dec 1;174(11):1221-8. Available from: https://www.ncbi.nlm.nih.gov/pubmed/16946126 
504 5. Rappold AG, Cascio WE, Kilaru VJ, Stone SL, Neas LM, Devlin RB, et al. Cardio-respiratory

505 outcomes associated with exposure to wildfire smoke are modified by measures of community

506 health. Environ Heal [Internet]. 2012;11(1):71. Available from: https://doi.org/10.1186/1476-

507 069X-11-71

508

509

510

511

512

513

514

515

516

517

518

519

520

521

522

523

524

525

526

6. Black C, Tesfaigzi Y, Bassein JA, Miller LA. Wildfire smoke exposure and human health: Significant gaps in research for a growing public health issue. Environ Toxicol Pharmacol [Internet]. 2017/08/30. 2017 Oct;55:186-95. Available from:

https://www.ncbi.nlm.nih.gov/pubmed/28892756

7. Liu JC, Pereira G, UhI SA, Bravo MA, Bell ML. A systematic review of the physical health impacts from non-occupational exposure to wildfire smoke. Environ Res [Internet]. 2014/11/20. 2015 Jan;136:120-32. Available from: https://www.ncbi.nlm.nih.gov/pubmed/25460628

8. Franzi LM, Bratt JM, Williams KM, Last JA. Why is particulate matter produced by wildfires toxic to lung macrophages? Toxicol Appl Pharmacol [Internet]. 2011/09/16. 2011 Dec 1;257(2):182-8. Available from: https://www.ncbi.nlm.nih.gov/pubmed/21945489

9. Nakayama Wong LS, Aung HH, Lamé MW, Wegesser TC, Wilson DW. Fine particulate matter from urban ambient and wildfire sources from California's San Joaquin Valley initiate differential inflammatory, oxidative stress, and xenobiotic responses in human bronchial epithelial cells. Toxicol Vitr [Internet]. 2011 Dec 1 [cited 2019 May 14];25(8):1895-905. Available from: https://www.sciencedirect.com/science/article/pii/S088723331100169X?via\%3Dihub

10. Verma V, Polidori A, Schauer JJ, Shafer MM, Cassee FR, Sioutas C. Physicochemical and Toxicological Profiles of Particulate Matter in Los Angeles during the October 2007 Southern California Wildfires. Environ Sci Technol [Internet]. 2009 Feb 1;43(3):954-60. Available from: https://doi.org/10.1021/es8021667 
527 11. Wegesser TC, Pinkerton KE, Last JA. California wildfires of 2008: coarse and fine particulate

matter toxicity. Environ Health Perspect [Internet]. 2009/02/02. 2009 Jun;117(6):893-7.

Available from: https://www.ncbi.nlm.nih.gov/pubmed/19590679

12. DeFlorio-Barker S, Crooks J, Reyes J, Rappold AG. Cardiopulmonary Effects of Fine Particulate Matter Exposure among Older Adults, during Wildfire and Non-Wildfire Periods, in the United States 2008-2010. Environ Health Perspect [Internet]. 2019 Oct 19;127(3):037006. Available from: https://doi.org/10.1289/EHP3860

13. Reid CE, Jerrett M, Tager IB, Petersen ML, Mann JK, Balmes JR. Differential respiratory health effects from the 2008 northern California wildfires: A spatiotemporal approach. Environ Res [Internet]. 2016 Oct 1 [cited 2019 Aug 8];150:227-35. Available from: https://www.sciencedirect.com/science/article/pii/S001393511630247X?via\%3Dihub

14. Delfino RJ, Brummel S, Wu J, Stern H, Ostro B, Lipsett M, et al. The relationship of respiratory and cardiovascular hospital admissions to the southern California wildfires of 2003. Occup Environ Med [Internet]. 2008/11/18. 2009 Mar;66(3):189-97. Available from: https://www.ncbi.nlm.nih.gov/pubmed/19017694

15. Spracklen D V, Mickley L, Logan JA, Hudman RC, Yevich R, Flannigan MD, et al. Impacts of climate change from 2000 to 2050 on wildfire activity and carbonaceous aerosol concentrations in the western United States. J Geophys Res Atmos [Internet]. 2009 Oct 27;114(D20). Available from: https://doi.org/10.1029/2008JD010966

16. Greenway J. CHIA data [Internet]. Center for Health Information Analysis, School of Public Health, University of Nevada. Las Vegas, NV.; 2018. Available from: https://chiaunlv.com/HealthFacilityData/AcquiringData_Services.php 
549 17. US Census Bureau. QuickFacts [Internet]. 2018 [cited 2019 Oct 26]. Available from: https://www.census.gov/quickfacts/fact/table/sparkscitynevada, renocitynevada/PST045218

18. Yang $\mathrm{W}$, Jennison B, Stanley O. AIR POLLUTION AND ASTHMA EMERGENCY ROOM VISITS IN RENO, NEVADA. Inhal Toxicol [Internet]. 1997 Jan 1;9(1):15-30. Available from: https://doi.org/10.1080/089583797198385

19. Rosenquist N, Metcalf W, Ryu S, Field-Ridley A, Coppes M, Grzymski J, et al. Acute associations 555

20. Wood S. Generalized Additive Models: An Introduction with R. 2nd ed. Boca Raton: Chapman \& Hall/CRC; 2017.

21. Clifford S. mgcv.helper: Helper Functions for mgcv. R package version 0.1.8. 2019.

22. Chan K-S, Ripley B. TSA: Time Series Analysis. R package version 1.2 [Internet]. 2018. Available from: https://cran.r-project.org/package=TSA

23. Dong TTT, Hinwood AL, Callan AC, Zosky G, Stock WD. In vitro assessment of the toxicity of bushfire emissions: A review. Sci Total Environ [Internet]. 2017 Dec 15 [cited 2019 Aug 6];603604:268-78. Available from:

https://www.sciencedirect.com/science/article/pii/S0048969717314663?via\%3Dihub

24. Kim YH, King C, Krantz T, Hargrove MM, George IJ, McGee J, et al. The role of fuel type and combustion phase on the toxicity of biomass smoke following inhalation exposure in mice. Arch Toxicol [Internet]. 2019;93(6):1501-13. Available from: https://doi.org/10.1007/s00204-01902450-5 
571 25. Abatzoglou JT, Kolden CA. Relative importance of weather and climate on wildfire growth in

572 interior Alaska. Int J Wildl Fire [Internet]. 2011;20(4):479-86. Available from:

$573 \quad$ https://doi.org/10.1071/WF10046

574 26. Shaposhnikov D, Revich B, Bellander T, Bedada GB, Bottai M, Kharkova T, et al. Mortality related 575

27. Li Y, Ma Z, Zheng C, Shang Y. Ambient temperature enhanced acute cardiovascular-respiratory mortality effects of PM2.5 in Beijing, China. Int J Biometeorol [Internet]. 2015;59(12):1761-70. Available from: https://doi.org/10.1007/s00484-015-0984-z

28. Ren C, Tong S. Temperature modifies the health effects of particulate matter in Brisbane, Australia. Int J Biometeorol [Internet]. 2006;51(2):87-96. Available from: https://doi.org/10.1007/s00484-006-0054-7

29. Roberts S. Interactions between particulate air pollution and temperature in air pollution 585 mortality time series studies. Environ Res [Internet]. 2004 Nov 1 [cited 2019 May 29];96(3):32837. Available from: https://www.sciencedirect.com/science/article/pii/S0013935104000222

30. Tian L, Liang F, Guo Q, Chen S, Xiao S, Wu Z, et al. The effects of interaction between particulate 588 matter and temperature on mortality in Beijing, China. Environ Sci Process Impacts [Internet]. 2018;20(2):395-405. Available from: http://dx.doi.org/10.1039/C7EM00414A mortality in Rome, Italy. Occup Environ Med [Internet]. 1998 Sep;55(9):605-10. Available from: https://www.ncbi.nlm.nih.gov/pubmed/9861182 
593 32. Lipner EM, O'Dell K, Brey SJ, Ford B, Pierce JR, Fischer EV, Crooks JL. The Associations Between 594 Clinical Respiratory Outcomes and Ambient Wildfire Smoke Exposure Among Pediatric Asthma 595 Patients at National Jewish Health, 2012-2015. GeoHealth. 2019; 3:146-59. Available from:

33. Gan RW, Liu J, Ford B, O'Dell K, Vaidyanathan A, Wilson A, et al. The association between wildfire smoke exposure and asthma-specific medical care utilization in Oregon during the 2013 wildfire season. Journal of Exposure Science \& Environmental Epidemiology. 2020; 30:618-28. Available 600 from: https://doi.org/10.1038/s41370-020-0210-x

34. US Environmental Protection Agency. Integrated Science Assessment for Particulate Matter (Final 602 Report). Washington, DC; 2009.

35. Leonard SS, Castranova V, Chen BT, Schwegler-Berry D, Hoover M, Piacitelli C, et al. Particle sizedependent radical generation from wildland fire smoke. Toxicology [Internet]. 2007 Jul 1 [cited 2019 Aug 7];236(1-2):103-13. Available from: https://www.sciencedirect.com/science/article/pii/S0300483X07002284?via\%3Dihub

36. Jalava PI, Salonen RO, Hälinen Al, Penttinen $\mathrm{P}$, Pennanen $\mathrm{AS}$, Sillanpää $\mathrm{M}$, et al. In vitro inflammatory and cytotoxic effects of size-segregated particulate samples collected during longrange transport of wildfire smoke to Helsinki. Toxicol Appl Pharmacol [Internet]. 2006 Sep 15 [cited 2019 Aug 7];215(3):341-53. Available from: https://www.sciencedirect.com/science/article/pii/S0041008X06001062?via\%3Dihub

612 37. US Environmental Protection Agency. Air Quality System Data Mart [internet database] 613 [Internet]. [cited 2019 Mar 18]. Available from: https://www.epa.gov/airdata 
Tables

616 Table 1 - Daily mean, range, and standard deviation for asthma visits, PM, and weather factors.

\begin{tabular}{lcccccccc}
\hline & \multicolumn{2}{c}{ All days } & & \multicolumn{2}{c}{ Non-wildfire days } & & \multicolumn{2}{c}{ Wildfire days } \\
\cline { 2 - 3 } Variable & mean (range) & SD & & mean (range) & SD & & mean (range) & SD \\
\hline Asthma visits & $8.6(0-25)$ & 3.9 & & $8.6(0-25)$ & 3.8 & & $8.2(1-21)$ & 3.9 \\
24-hr PM 2.5 $\left(\mu \mathrm{g} / \mathrm{m}^{3}\right)$ & $8.1(0.1-99.9)$ & 7.2 & & $7.1(0.1-40.4)$ & 4.8 & & $19.4(3.9-99.9)$ & 14.8 \\
24-hr PM 10-2.5 $\left(\mu \mathrm{gg} / \mathrm{m}^{3}\right)$ & $11.0(0.4-60.8)$ & 6.9 & & $10.9(0.4-60.8)$ & 7.1 & & $11.6(3.2-40.3)$ & 4.8 \\
24-hr PM 10 $\left(\mu \mathrm{gg} / \mathrm{m}^{3}\right)$ & $19.0(2.0-118.3)$ & 11.9 & & $17.9(2.0-86.3)$ & 10.6 & $31.2(10.3-118.3)$ & 16.5 \\
Mean temperature $\left({ }^{\circ} \mathrm{C}\right)$ & $13.3(-12.2-33.1)$ & 9.1 & & $12.2(-12.2-33.1)$ & 8.7 & $24.4(10.9-31.7)$ & 4.0 \\
Mean relative humidity $(\%)$ & $43.0(12.3-92.3)$ & 17.1 & $44.3(12.3-92.3)$ & 17.1 & $29.2(15.3-88.8)$ & 9.7 \\
\hline SD:
\end{tabular}

617

SD: standard deviation

618

619

620

621

622

623

624

625

626

627

628

629

630 
631 Table 2 - Pearson correlations between daily averages of PM and weather factors.

\begin{tabular}{|c|c|c|c|c|c|c|c|c|c|c|c|c|}
\hline \multirow[b]{2}{*}{ Variable } & \multicolumn{4}{|c|}{ All days } & \multicolumn{4}{|c|}{ Non-wildfire days } & \multicolumn{4}{|c|}{ Wildfire days } \\
\hline & $\mathrm{PM}_{10-2.5}$ & $\mathrm{PM}_{10}$ & Temp & RH & $\mathrm{PM}_{10-2.5}$ & $\mathrm{PM}_{10}$ & Temp & RH & $\mathrm{PM}_{10-2.5}$ & $\mathrm{PM}_{10}$ & Temp & RH \\
\hline $\mathrm{PM}_{2.5}$ & 0.45 & 0.86 & 0.00 & 0.05 & 0.67 & 0.87 & -0.30 & 0.28 & 0.19 & 0.96 & -0.05 & 0.03 \\
\hline $\mathrm{PM}_{10-2.5}$ & & 0.84 & -0.15 & 0.01 & & 0.94 & -0.17 & 0.02 & & 0.46 & -0.30 & 0.05 \\
\hline $\mathrm{PM}_{10}$ & & & -0.07 & 0.03 & & & -0.23 & 0.13 & & & -0.14 & 0.04 \\
\hline Temp & & & & -0.78 & & & & -0.78 & & & & -0.44 \\
\hline
\end{tabular}

632 RH: daily mean relative humidity, Temp: daily mean temperature.

633

634

635

636

637

638

639

640

641

642

643

644

645

646 
648 Figure 1-Map of the Reno-Sparks area showing the locations of air quality monitors, urgent care 649 centers, emergency departments, and the KRNO weather station.

650 Figure 2 - Time series of particulate matter and asthma visits from January 1, 2013, to December 31, 6512018 in Reno, Nevada. Daily values of $\mathrm{PM}_{2.5}$ and $\mathrm{PM}_{10-2.5}$ are weighted averages across all air quality 652 monitors. The width of the blue ribbon indicates the number of asthma visits, and the orange-shaded 653 regions below the time series for $\mathrm{PM}_{2.5}$ indicate days where the air quality was determined by WCHD-

$654 \quad A Q M D$ to be affected by wildfire smoke.

655 Figure 3 - Rate ratios for asthma visits to the ED and UC centers in the presence or absence of wildfire 656 smoke. PM was scaled to $5 \mu \mathrm{g} / \mathrm{m}^{3}$ for all fractions and lags. Numeric labels between points are

657 estimates of the change in the rate ratio when wildfire smoke is present compared to when it is not 658 present, presented as a factor. This change can also be presented as a percent (i.e., the factor 1.028 is 659 equivalent to an increase of $2.8 \%$ ), which is how the changes are presented in the body of the paper.

660 Rate ratios and the differences between them are presented in tabular form in Table S1.

661 Figure 4 - Percent increase in asthma visits due to wildfire smoke, at given levels of $\mathrm{PM}_{2.5}$ and $\mathrm{PM}_{10-2.5}$.

662 Estimates were constrained to the regions within $5 \mu \mathrm{g} / \mathrm{m}^{3}$ of a wildfire data point to limit extrapolation. 663 Shaded regions indicate estimates that were significant at the 0.05 level. 
Figures

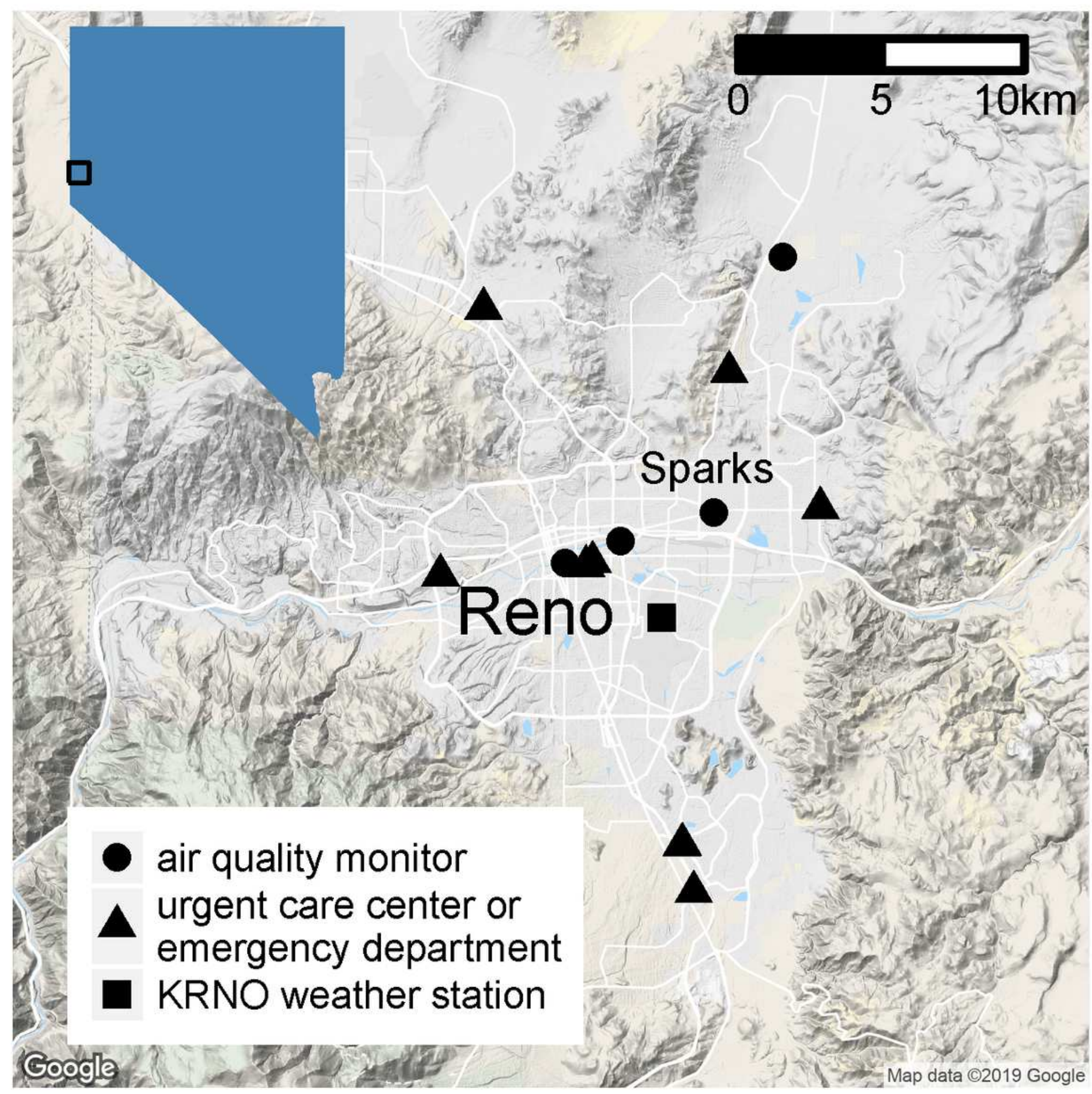

Figure 1

Map of the Reno-Sparks area showing the locations of air quality monitors, urgent care centers, emergency departments, and the KRNO weather station. 

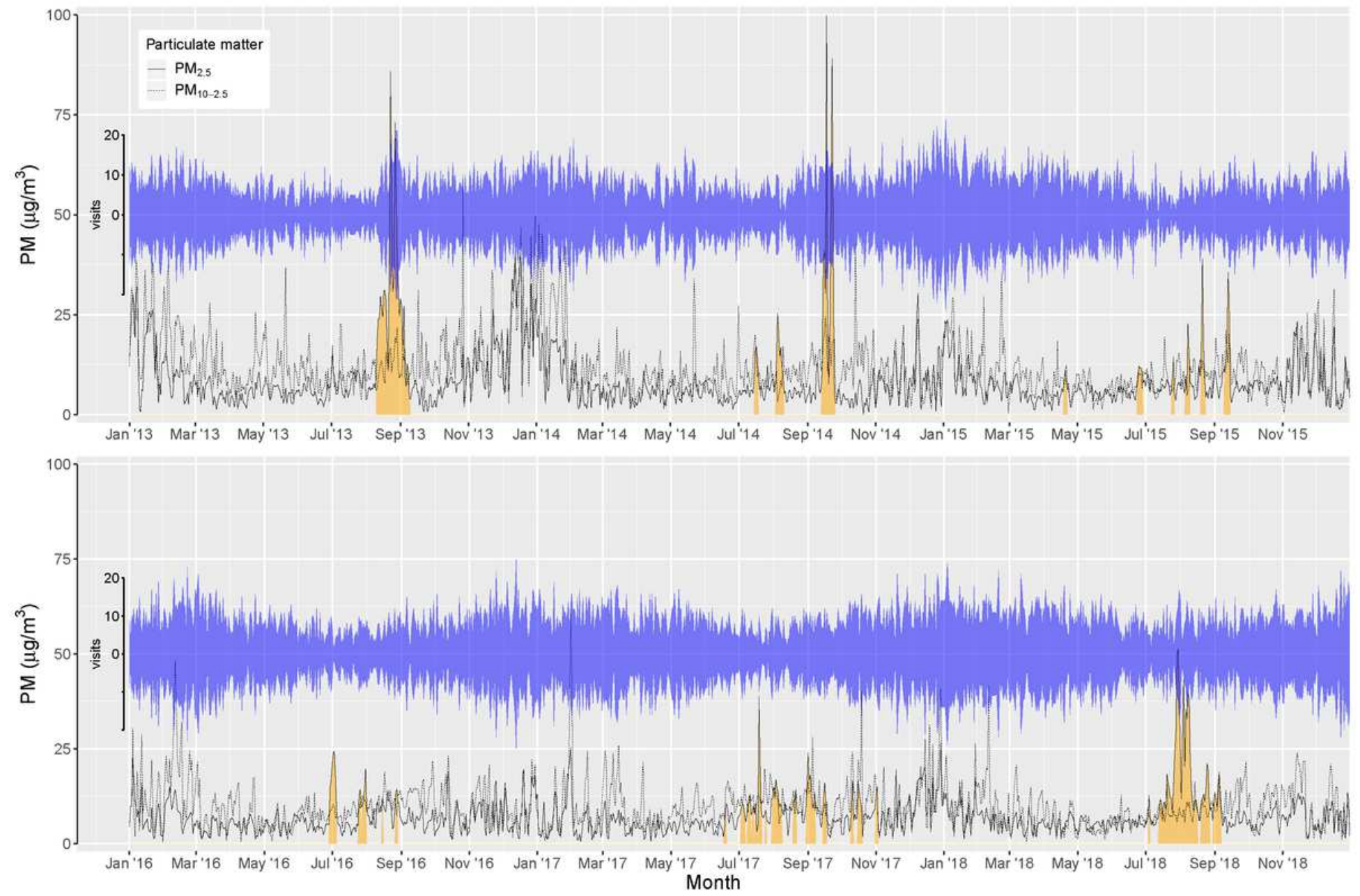

\section{Figure 2}

Time series of particulate matter and asthma visits from January 1, 2013, to December 31, 2018 in Reno, Nevada. Daily values of PM2.5 and PM10-2.5 are weighted averages across all air quality monitors. The width of the blue ribbon indicates the number of asthma visits, and the orange-shaded regions below the time series for PM2.5 indicate days where the air quality was determined by WCHD-AQMD to be affected by wildfire smoke. 


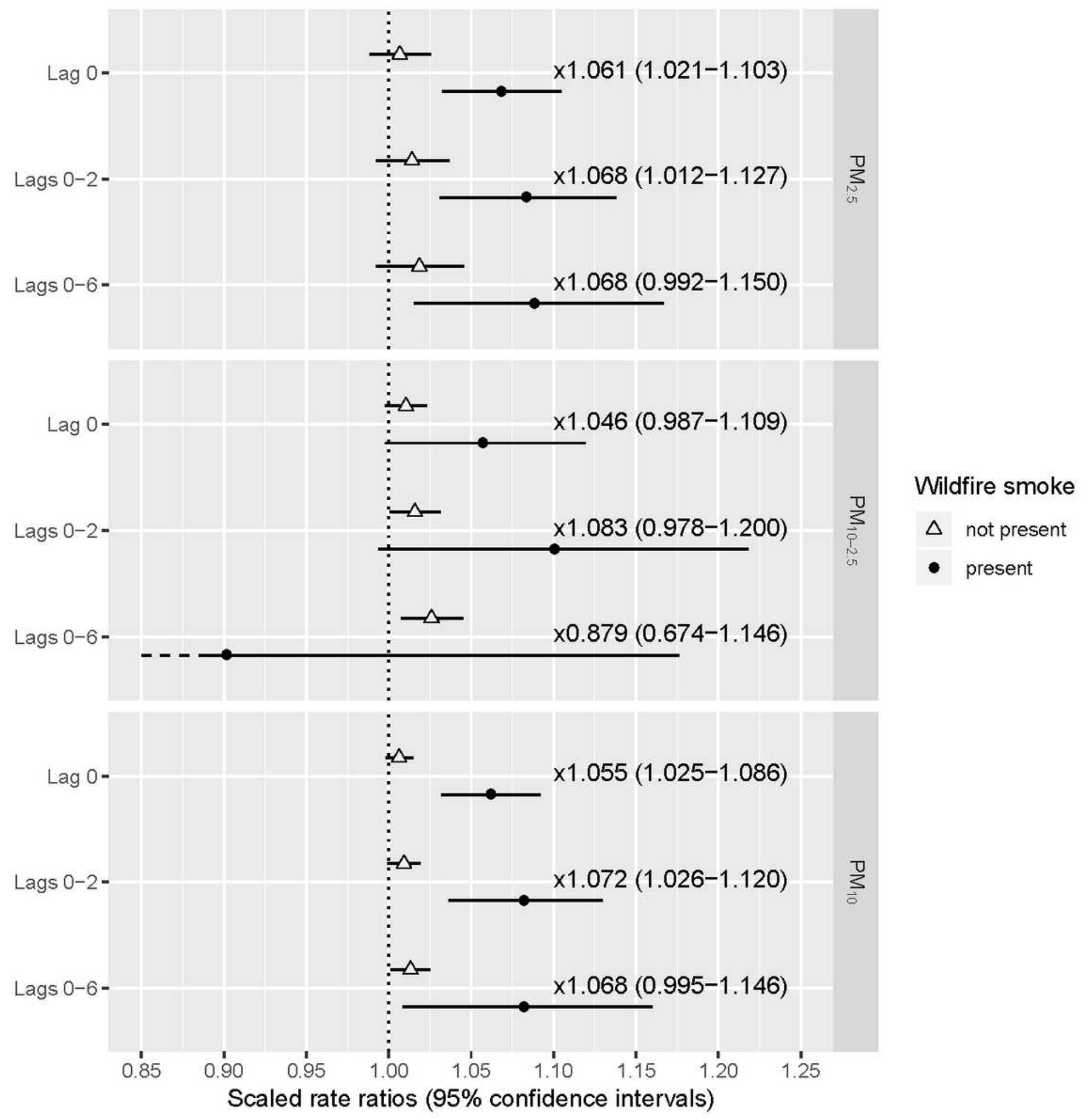

\section{Figure 3}

Rate ratios for asthma visits to the ED and UC centers in the presence or absence of wildfire smoke. PM was scaled to $5 \mu \mathrm{g} / \mathrm{m} 3$ for all fractions and lags. Numeric labels between points are estimates of the change in the rate ratio when wildfire smoke is present compared to when it is not present, presented as a factor. This change can also be presented as a percent (i.e., the factor 1.028 is equivalent to an increase of $2.8 \%$ ), which is how the changes are presented in the body of the paper. Rate ratios and the differences between them are presented in tabular form in Table S1. 


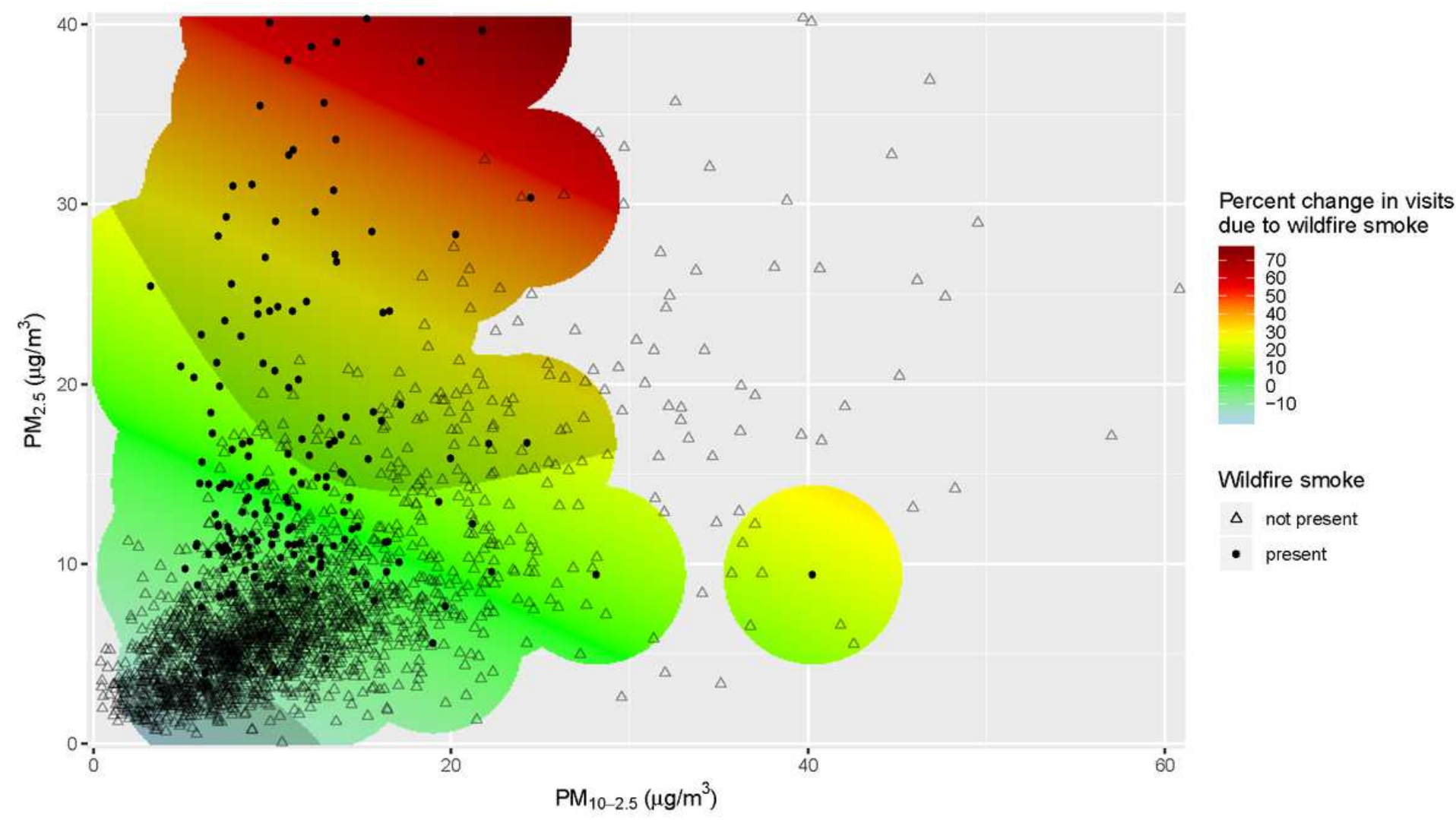

Figure 4

Percent increase in asthma visits due to wildfire smoke, at given levels of PM2.5 and PM10-2.5.

Estimates were constrained to the regions within $5 \mu \mathrm{g} / \mathrm{m} 3$ of a wildfire data point to limit extrapolation. Shaded regions indicate estimates that were significant at the 0.05 level.

\section{Supplementary Files}

This is a list of supplementary files associated with this preprint. Click to download.

- SupplementalMaterial102319revised.docx 